\title{
Anemia em população de área endêmica de malária, Rondônia (Brasil)*
}

\author{
Anaemia in a population sample from an endemic malaria area of Rondônia \\ State, Brazil
}

Marly A. Cardoso*, Marcelo U. Ferreira , Luis M. Aranha Camargo , Sophia C. Szarfarc

CARDOSO, M.A.. et al. Anemia em população de área endêmica de malária, Rondônia (Brasil). Rev. Saúde públ., S. Paulo, 26: 161-6, 1992. Com o objetivo de descrever a prevalência de anemia na população de uma área endêmica de malária - o distrito de Candeias, localidade periurbana do Município de Porto Velho, no Estado de Rondônia, Amazônia Ocidental Brasileira, - uma amostra casual de 1.068 indivíduos (14,1\% da população total) de todas as idades submeteu-se à dosagem de hemoglobina e à microscopia em gota espessa para diagnóstico de malária. O diagnóstico de anemia foi positivo em 299 indivíduos $(28,0 \%$ da amostra), sendo as maiores prevalências encontradas em crianças com idade entre 6 meses e um ano $(70,0 \%)$ e entre um e 6 anos $(38,4 \%)$, além de gestantes $(41,2 \%), 7 / 17)$ e pacientes com malária $(44,4 \%$, 8/18). Realizaram-se exames parasitológicos de fazes em amostra voluntária de 476 indivíduos (44,6\% da população amostral), dos quais $118(26,3 \%)$ foram positivos. Nessa amostra voluntária, não houve diferença significante na prevalência de anemia entre individuos parasitados e não-parasitados. Acima de 14 anos de idade, a prevalência de anemia foi tanto maior quanto mais recente o último episódio de malária referido pelos pacientes. Nesta faixa etária, economicamente produtiva, destaca-se o papel da malária entre as causas subjacentes à anemia.

Descritores: Anemia, epidemiologia. Malária, complicações. Enteropatias parasitárias, complicações.

\section{Introdução}

O controle da malária e o combate à anemia estão entre os programas prioritários da Organização Mundial da Saúde (OMS). Em 1980, a OMS estimava a existência de 700 milhões de individuos anêmicos em todo o mundo, com importantes repercussões sobre seu desenvolvimento físico e mental ${ }^{5}$. A deficiência de ferro na alimentação tem sido apontada como a determinante causal principal da esmagadora maioria dos casos, tornando-a a carência nutricional mais prevalente no mundo atual ${ }^{5,9,23}$.

A malária está entre as doenças parasitárias mais prevalentes nos paises tropicais, estimandose sua ocortência anual de 210 a 220 milhões de $\operatorname{casos}^{19,22,33}$. No Brasil, a Fundação Nacional de Saúde (FNS), órgão do Ministério da Saúde,

* Realizado com apoio financeiro do Conselho Nacional de Desenvolvimento Científico e Tecnológico (CNPq) - (Processo 403404/90-8).

** Núcleo de Pesquisas Epidemiológicas em Nutrição e Saúde (NUPENS) da Universidade de São Paulo (USP) - Săo Paulo - Brasil.

*** Departamento de Parasitologia do Instituto de Ciências Biomédicas da USP e NUPENS.

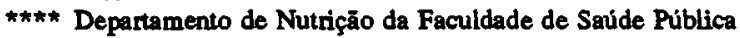
da USP e NUPENS.

Separatas/Reprints: S.C. Szarfarc - Av. Dr. Amaldo, 715 01246-904 - São Paulo, SP - Brasil

Publicação financiada pela FAPESP. Processo Saúde Coletiva 91/4994-0 registrou 577 mil casos novos de malária em 1989, dos quais 97\% diagnosticados na Região Amazônica. O Estado de Rondônia, que reúne cerca de $1 \%$ da população do país, notificou 42,4\% dos casos diagnosticados no Brasil, naquele ano ${ }^{13}$.

Anemia e malária coexistem em diversas regiōes do mundo. A malária é causa importante de anemia hemolítica, durante a doença aguda e na fase de recuperação da infecção, quando parecem atuar mecanismos auto-imunes ainda pouco esclarecidos ${ }^{32}$. Nas relaçōes entre anemia $e$ malária, outro aspecto importante a ser destacado refere-se à possível proteção relativa conferida pela anemia ferropriva à malária em áreas holoendêmicas ${ }^{17}$. Os parasitas do gênero Plasmodium instalam-se nos eritrócitos, provocando hemólise e utilizando-se de ferro-heme para sua nutrição. $O$ uso de quelantes de ferro em culturas "in vitro" de $P$. falciparum inibe o crescimento do parasita ${ }^{8}$. Dados provenientes da África Ocidental sugerem que indivíduos portadores de $P$. falciparum, com anemia ferropriva, têm menor susceptibilidade às manifestações clínicas da malária, embora possam surgir sintomas de parasitemia após a correção da anemia ${ }^{15,16}$. Este fenômeno foi observado em Papua Nova Guiné, entre lactentes que receberam suplementação de ferro por via parenteral ${ }^{18}$; resultado discordante foi, no entanto, observado por McGregor ${ }^{12}$ com administração de ferto por via oral. 
O presente trabalho destina-se a investigar um dos aspectos das relações entre anemia e malária apontados aqui, ou seja, descrever a prevalência de anemia em indivíduos habitantes de uma área endêmica de malária e discutir o possível impacto da malária sobre a epidemiologia da anemia nessa população.

\section{Material e Método}

\section{Área estudada e populaçāo}

O estudo foi realizado em Candeias do Jamari, distrito do Município de Porto Velho, capital do Estado de Rondônia. O distrito situa-se a $21 \mathrm{~km}$ a leste de Porto Velho, às margens do Rio Candeias, cerca de $85 \mathrm{~m}$ acima do nível do mar. Candeias é exemplo dos aglomerados populacionais que vêm se desenvolvendo em torno das cidades da Amazônia Brasileira. A área urbana reúne 2.235 domicílios, dos quais cerca de $30 \%$ (cujos proprietários moram em Porto Velho ou em sítios nos arredores de Candeias) permanecem desocupados a maior parte do ano. A metade dos domicílios dispõe de água encanada e $70 \%$ deles são ligados à rede de fornecimento de energia elétrica. Não existe rede de esgotos. Censo populacional efetuado pela FNS em 1990 estima a existência de cerca de 7.600 habitantes na área urbano. Naquele ano, o posto local da FNS diagnosticou 1.340 casos novos de malária (média mensal de 112 casos), dos quais 466 $(34,8 \%)$ por $P$. falciparum e $869(64,9 \%)$ por $P$. vivax, além de 5 casos $(0,4 \%)$ de malária mista. A maioria dos casos $(64,5 \%)$ ocorreu em indivíduos do sexo masculino. Observou-se variação sazonal no número de casos de malária notificados em Candeias ao longo de 1990: a maior incidência ocorreu em maio, junho e julho.

\section{Amostragem}

Uma amostra aleatória sistemática de 9,6\% dos domicílios de Candeias foi visitada, sendo colhidas amostras sangüíneas dos habitantes presentes no momento da visita, perfazendo 1.068 indivíduos de todas as idades $(14,1 \%$ da população total do distrito), independente da condição de saúde ou estado físiológico*.

* Esta amostragem foi elaborada pelos professores D. Sawyer (UFMG) e P.L. Tauil (UnB) e utilizada em estudo preliminar sobre características sócioeconómicas do distrito de Candeias (dados não-publicados).

\section{Coleta e processamento de amostras}

As atividades de campo realizaram-se entre os meses de agosto e novembro de 1990, antes do início da estação chuvosa.

Após consentimento informado (de cada paciente ou de seus responsáveis), os indivíduos foram submetidos à punção venosa periférica para coleta de amostras sangüíneas. As amostras destinaram-se a: a) lâmina com duas gotas espessas para pesquisa de plasmódio; b) tubo heparinizado (aproximadamente $1 \mathrm{ml}$ de sangue), para dosagem de hemoglobina e determinação de hematócrito; e c) tubo seco (aproximadamente $9 \mathrm{ml}$ de sangue), para separação de soro a ser empregado em exames imunodiagnósticos. Simultaneamente, todos os indivíduos foram submetidos a entrevista e exame clínico. As amostras fecais, fornecidas voluntariamente por 476 indivíduos $(44,6 \%$ do total), foram acondicionadas em recipientes plásticos com tampa.

As amostras sangüíneas foram processadas, até cinco horas após a coleta, no Laboratório de Pesquisa em Malária da Universidade de São Paulo, instalado no Centro de Hematologia e Hemoterapia de Rondônia (HEMERON), em Porto Velho. As amostras fecais foram conservadas a $4^{\circ} \mathrm{C}$ para posterior exame (até 15 dias após a coleta).

Para dosagem de hemoglobina empregou-se o método da cianometa-hemoglobina ${ }^{6}$, com padrão artificial de calibração ${ }^{31}$, estável por longos periodos. Os resultados de hematócrito, obtidos pela técnica rotineiramente utilizada ${ }^{7}$, foram empregados como controle da dosagem de hemoglobina. O quociente do valor de hematócrito (em \%), dividido pela concentração de hemoglobina (em $\mathrm{g} / 100 \mathrm{ml}$ ), distribuiu-se gaussianamente em torno de 2,9. Sempre que o quociente hematócrito/hemoglobina foi inferior a 2,4 ou superior a 3,4 repetiram-se as dosagens de hemoglobina.

O diagnóstico de malária foi feito mediante o exame microscópico de gota espessa corada pela técnica de Walker ${ }^{21}$.

Com o objetivo de verificar o papel das parasitoses intestinais na etiologia da anemia, realizaram-se exames parasitológicos de fezes por método direto ${ }^{1}$, sem avaliação quantitativa.

Todos os pacientes em que se diagnosticou anemia, malária e/ou doença parasitária intestinal receberam medicação adequada, segundo prescrição dos profissionais médicos da equipe de pesquisa de campo. 


\section{Resultados}

$\mathrm{Na}$ amostra estudada predominaram indivíduos pardos $(59,6 \%)$, sendo $37,2 \%$ brancos e $3,2 \%$ negros; a maioria $(71,8 \%)$ provinha da Região Norte e $14,0 \%$ eram nordestinos. Cerca de $86 \%$ dos individuos entrevistados residiam em Candeias há menos de 5 anos.

Empregaram-se os valores-limite de concentração de hemoglobina estabelecidos pela OMS para a definição de anemia nas faixas etárias acima de 6 meses de idade ${ }^{20}$. Com este critério, diagnosticou-se anemia em 299 indivíduos (28,0\% do total), residentes em 133 domicílios $(61,9 \%$ do total). Em crianças com até 6 meses de idade ( 7 indivíduos na amostra), o diagnóstico de anemia pode basear-se nos valores médios de concentração de hemoglobina esperados ao longe desse periodo ${ }^{4}$. Seis crianças nesta faixa etária apresentaram valores de concentração de hemoglobina abaixo dos valores médios esperados. A Tabela 1 mostra o número de indivíduos e a percentagem de anêmicos distribuídos por sexo e faixa etária. A proporção de indivíduos do sexo masculino com anemia foi significantemente maior (qui-quadrado $=8,11, p<0,01$ ). Das 17 gestantes amostradas, $7(41,2 \%)$ eram anêmicas. Dos 18 pacientes com malária diagnosticada durante o estudo ( 9 infecções por $P$. falciparum e 9 por $P$. vivax), $8(44,4 \%)$ tinham anemia. Encontrou-se anemia severa (concentração de hemoglobina sangüínea abaixo de $7 \mathrm{~g} / 100 \mathrm{ml}$ ) em 4 indivíduos, enviados para investigaçāo etiológica em serviço de saúde de nivel terciário.

Tabela 1. Número de individuos ( $n$ ) e percentagem de anémicos $(\% \mathrm{~A})$ segundo sexo e faixa etária na amostra populacional (1.068 indivíduos) do distrito de Can. deias, Estado de Rondônia.

\begin{tabular}{lrrrrr}
\hline & \multicolumn{3}{c}{ Sexo } & \\
\cline { 2 - 5 } $\begin{array}{l}\text { Faixa } \\
\text { etária (anos) }\end{array}$ & Feminino & Masculino & $\begin{array}{c}\text { Ambos os } \\
\text { sexos }\end{array}$ \\
\hline$<0,5$ & $3(100,0)$ & $4(75,0)$ & $7(85,7)$ \\
$0,5 \cdot 1$ & 6 & $(83,3)$ & $14(64,3)$ & $20(70,0)$ \\
$2 \cdot 5$ & $139(36,7)$ & $140(40,0)$ & $279(38,4)$ \\
$6 \cdot 14$ & 130 & $(25,4)$ & 158 & $(35,4)$ & $288(30,9)$ \\
$>14$ & 299 & $(17,4)$ & 175 & $(21,1)$ & $474(18,8)$ \\
\hline Total & 574 & $(24,4)$ & 487 & $(32,2)$ & $1.068(28,8)$ \\
\hline
\end{tabular}

Da população amostral, 700 indivíduos $(65,5 \%)$ referiram ter tido malária pelo menos uma vez, contraída ou não em Candeias. A Tabela 2 mostra a prevalência de anemia segundo
Tabela 2. Prevaléncia de anemia segundo história de malária prévia o faixa etária, na amostra populacional (1.068 individuos) do distrito de Candeias, Estado de Rondónia.

\begin{tabular}{lcccc}
\hline & \multicolumn{2}{c}{$\begin{array}{c}\text { Sem malária } \\
\text { prévia }\end{array}$} & \multicolumn{2}{c}{$\begin{array}{c}\text { Com malária } \\
\text { prévia }\end{array}$} \\
\cline { 2 - 5 } $\begin{array}{l}\text { Faixa etária } \\
\text { (anos) }\end{array}$ & $n$ & $\%$ anêmicos & $n$ & $\%$ anémicos \\
\hline $0,5 \cdot 1$ & 25 & 72,0 & 2 & 50,0 \\
$1 \cdot 6$ & 166 & 37,4 & 113 & 39,8 \\
$6 \cdot 14$ & 90 & 35,6 & 198 & 28,8 \\
$>14$ & 87 & 18,4 & 387 & $18,8 \ldots$ \\
\hline Total & 368 & 34,8 & 700 & 25,1 \\
\hline
\end{tabular}

história de malária prévia, esta segundo informação dos pacientes ou de seus responsáveis, distribuida segundo faixas etárias, entre os 1.068 indivíduos da população amostral. Em nenhuma faixa etária houve diferença significativa na prevalência de anemia entre indivíduos que referem e os que negam passado malárico. Entretanto, quando se analisam os pacientes que referem passado malárico quanto ao tempo decorrido desde o último episódio (Figura 1), evidencia-se tendência de queda da prevalência de anemia à medida que se distancia o último episódio malárico. Esta tendência é significante entre indivíduos com idade superior a 14 anos (qui-quadrado de tendência ${ }^{11}=12,909, \mathrm{p}<0,001$ ) porém não entre individuos de idade igual ou inferior a esta (qui-quadrado de tendência $=1,106,0,20<\mathrm{p}<$ $0,30)$.

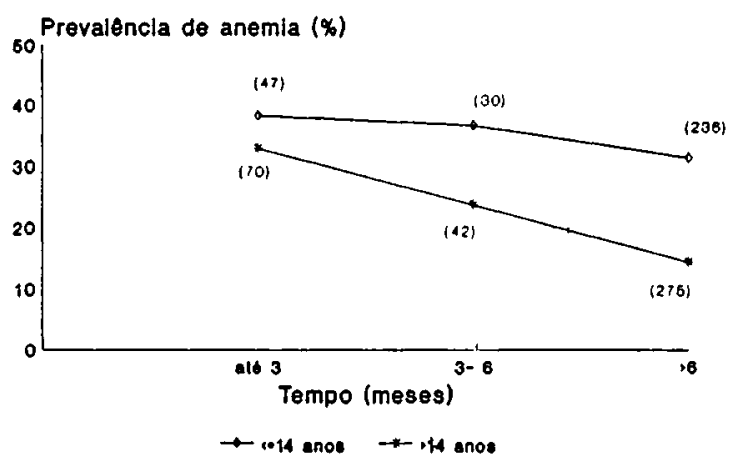

Figura 1. Prevaléncia de anemia segundo o tempo decorrido desde o último episódio de malária, entre os individuos da populaçáo amostral do distrito de Candelas que referem passado malárico $(n=700)$. A amos. tra foi distribuida em duas faixas etárias: a) idade igual ou inferior a 14 anos $(n-313)$; e b) idade superior a 14 anos $(n-387)$. Os números entre parênteses mostram o total de individuos em cada grupo. 


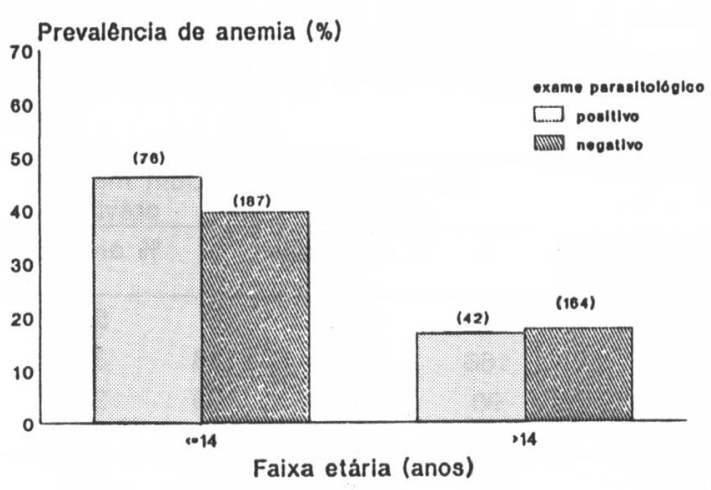

Figura 2. Prevaléncia de anemia segundo faixa etária entre os 476 indivíduos de Candelas submetidos a exame parasitológico de fezes durante o estudo. Os números entre parénteses indicam 0 total de indivíduos em cada coluna.

A Figura 2 mostra a prevalência de anemia entre indivíduos submetidos a exame parasitológico de fezes, distribuídos em dois grupos: a) idade igual ou inferior a 14 anos; e b) idade superior a 14 anos. Em nenhum dos grupos houve diferença significante na prevalência de anemia entre indivíduos com exame parasitológico de fezes positivo e negativo: a) qui-quadrado = $0,985,0,30<p<0,50 ;$ b) qui-quadrado $=0,011$, $0,90<\mathrm{p}<0,95$. Do total de 476 exames realizados, $118(24,8 \%)$ foram positivos. A Tabela 3 mostra frequiência de positividade para as espécies parasitárias mais comumente encontradas nesses exames. Ovos de Ancylostomidae, os parasitas intestinais humanos mais freqüentemente associados à anemia, foram detectados em 27 amostras $(5,7 \%)$, das quais 7 pertenciam a indivíduos anêmicos.

Tabela 3. Freqüência absoluta e relativa (\%) de amostras fecais positivas para parasitas intestinais entre 476 individuos da populaçấo amostral do distrito de Candeias, Estado de Rondónia.

\begin{tabular}{ccc}
\hline & \multicolumn{2}{c}{ Amostras positivas } \\
\cline { 2 - 3 } Parasita intestinal & $\mathrm{n}$ & $\%$ \\
\hline Ascaris lumbricoides & 67 & 14,1 \\
Hymenolepis nana & 29 & 6,1 \\
Ancylostomidae & 27 & 5,7 \\
Outros & 8 & 1,7 \\
\hline Total de amostras & 118 & 24,8 \\
positivas * & & \\
\hline
\end{tabular}

- Em 18 amostras fecais mais de uma espécie para. sitária fol encontrada

\section{Discussão}

Em áreas endêmicas de malária, três fatores (muitas vezes sobrepostos) destacam-se na etiologia da anemia: deficiência nutricional, parasitoses intestinais e malária.

A anemia nutricional é um inquestionável problema de saúde pública no Brasil. Becker \& Lechtig ${ }^{2}$, através de dados da Divisão Nacional de Epidemiologia, concluíram que cerca de 14 mil óbitos $(1,3 \%$ do total) entre a população infantil brasileira são causados direta ou indiretamente pela anemia, sendo esta responsável também por cerca da metade dos óbitos em menores de 5 anos e em mulheres em idade fértil na região Nordeste do Brasil.

O Instituto Nacional de Alimentação e Nutrição (INAN) propôs em 1987 um programa de controle de anemia em todo o território nacional. Em Rondônia seriam beneficiários do programa 16 mil crianças de 6 a 36 meses de idade e 92 mil mulheres gestantes e nutrizes.

A escassez de dados epidemiológicos sobre a anemia no Brasil dificulta a análise comparativa dos resultados aqui apresentados. A maioria dos estudos disponiveis refere-se exclusivamente a crianças ${ }^{10,25,26}$ e a gestantes ${ }^{24,28,29,30}$, considerados os grupos de maior vulnerabilidade, ou a usuários de serviços de saúde ${ }^{25,29}$, que não compōem necessariamente uma amostra representativa da população geral. São ainda mais escassos estudos relativos à Amazônia Brasileira que incluam na população amostral adultos do sexo masculino, grupo mais exposto ao risco de transmissão de malária.

A maior prevalência de anemia em crianças, observada no presente estudo (Tabela 1), é também referida por outros pesquisadores ${ }^{14,25,26}$. Em geral, verifica-se prevalências maiores na população da faixa etária entre 6 meses e 2 anos. A maior requisiçāo de ferto no primeiro ano de vida $(120 \mathrm{ug} / \mathrm{kg}$ de peso/dia) e sua diminuiçāo nas idades pré-escolar e escolar (50 e $40 \mathrm{ug} / \mathrm{kg}$ de peso/dia, respectivamente) ${ }^{5}$ explicam a prevalência de anemia nesses grupos (Tabela 1). No entanto, as diferenças fisiológicas de necessidades de ferro em maiores de 14 anos - respectivamente 18 e $45 \mathrm{ug} / \mathrm{kg}$ de peso/dia para homens e mulheres ${ }^{5}$ - não explicam a maior ocortência de anemia entre homens na população estudada, sugerindo a influência de outros fatores de expoliação de ferro e demais nutrientes neste grupo.

A anemia por parasitismo intestinal associase comumente à infeç̧ão por Ancylostomidae. Embora a anemia decorrente desta infecção seja 
em geral mais intensa que a associada à ascaríase, a maioria dos helmintos intestinais pode produzir doença severa no caso de cargas parasitárias elevadas ${ }^{27}$. No estudo de Candeias, a ausência de dados quantitativos (contagens de ovos nas fezes ou de vermes expulsos após o tratamento) impossibilita inferências sobre as relações entre os niveis de hemoglobina e a intensidade de infecção parasitária. Entretanto, a distribuição homogênea da anemia entre indivíduos parasitados e não-parasitados, que compunham a amostra voluntária em que se realizaram exames coproparasitológicos, sugere que a infecção por parasitas intestinais não esteja entre os principais determinantes causais de anemia na população amostral de Candeias.

A Amazônia Brasileira é caracteristicamente uma área de transmissão instável de malária. Neste contexto epidemiológico, que difere por exemplo da situação africana, a transmissão de malária restringe-se em geral às bordas das florestas, sendo menos intensa em povoados e cidades. Por isso, caracterizam-se grupos com maior exposição - agricultores, garimpeiros e construtores de estradas - compostos principalmente por indivíduos do sexo masculino em idade produtiva ${ }^{19}$. Embora a malária em Candeias acometa indivíduos de qualquer idade, a exposiçāo dos adultos tende a ser maior, em função de suas atividades de trabalho. A Figura 2 mostra que, entre indivíduos com mais de 14 anos, episódios recentes de malária são associados, de modo significante, a maior prevalência de anemia, sendo necessários pelo menos três meses para que os indivíduos com um episódio malárico readquiram niveis de hemoglobina semelhantes aos da população getal. A recuperação dos parâmetros hematológicos após um episódio malárico ocorre em períodos de tempo muito variáveis, dependendo, entre outros, das reservas orgânicas individuais de ferro ${ }^{3,5}$. No entanto, é provável que um intervalo de tempo muito maior decorra até que as reservas orgânicas de ferto - e de outros nutrientes relacionados à síntese de hemoglobina - estejam plenamente repostas nesses pacientes que, nessa situação, se tornam particularmente vulneráveis à anemia.

\section{Agradecimentos}

Aos professores Emey Plessmann Camargo (USP) e Luiz Hildebrando Pereira da Silva (Instituto Pasteur de Paris), responsáveis pelas pesquisas sobre malária em Rondônia desenvolvidas pelo Instituto de Ciências Biomédicas da USP, pela infra-estrutura cedida; ao Dr. Francisco Roberto dos Santos, diretor geral do Centro de Hematologia e Hemoterapia de Rondônia (HEMERON), pelas inúmeras sugestões técnicas; à Teresinha Luisa Dinon (SESAU), diretora administrativa do HEMERON, à Anny M.B. de Camargo Costa e à Cleonice da Silva Cavalcante, pela participação nas atividades de campo e laboratoriais.

CARDOSO, M.A et al. [Anaemia in a population sample from an endemic malaria area of Rondónia State, Brazil. Rev. Saúde públ., S. Paulo, 26: 161-6, 1992. With the purpose of describing the prevalence rate of anaemia among inhabitants of a malaria endemic area - Candeias district, a periurban locality near Porto Velho, in Rondônia State, Brazilian Amazon Basin - a random population sample comprehending 1,068 individuals of all age groups (14.1\% of the total population) was screened for anaemia (measurement of blood haemoglobin concentration) and malaria (Giemsa-stained thick-smear microscopy).

Two-hundred and ninety-nine individuals $(28.0 \%$ of the sample) were found to be anaemic, using the cut-off haemoglobin values proposed by the World Health Organization for each age group. Highest prevalence rates were found among children with ages varying from 6 months to 1 year $(70.0 \%)$ and from 1 to 6 years $(38.4 \%)$, as well as in pregnant women $(41.2 \%, 7 / 17)$ and malaria patients $(44.4 \%$, $8 / 18$ ). Parasitological stoll examinations were made on a voluntary sample of 476 individuals $(44.6 \%$ of the sample population); of these, $118(26.8 \%)$ were positive. Eggs of Ascaris lumbricoides, the most frequent intestinal parasite in this population sample, was detected in 67 stool samples (14.1\%); only 27 patients (5.7\%) eliminated Ancylostomidae eggs. In this voluntary sample, no significant difference in anaemia prevalence rates between parasite carriers and non-parasited individuals was detected. On the other hand, the more recent the last malarial episode referred to by the patients, the ligher prevalence rate of anaemia in individuals above the age of 14 years. The role played by malaria as an underlying cause of anaemia in Candeias district inhabitants, particularly in the economically active age group, is further discussed.

Keywords: Anemia, epidemiology. Malaria, complications. Intestinal diseases, parasitic, complications.

\section{Referências Bibliográficas}

1. BEAVER, P.C. et al. Examination of specimens for parasites. In: Beaver, P.C. et al. Clinical parasitology. 9th ed. Philadelphia, Lea \& Febiger, 1984. p. 733-64.

2. BECKER, N.A. \& LECHTIG, A. Brasil: evolução da mortalidade infantil no períado 1977-1984. Brasília, Ministério da Saúde, 1986. 
3. BRADLEY-MOORE, A.M. et al. Malaria chemoprophylaxis with chloroquine in young Nigerian children. IV - Its effect on haematological measurements. Ann. trop. Med. Parasit., 79: 585-95, 1985.

4. BRAULT-DUBUC, M. et. al. Iron status of French-Canadian children: a three year follow-up study. Hum. Nutr. Appl. Nutr., 37A: 210-21, 1983.

5. De MAYER, E.M. et al. Preventing and controlling iron deficiency anemia through primary health care. Geneva, World Health Organization, 1989.

6. DRABKIN, D.L. \& AUSTIN, S.H. Spectrophotometric studies. II - Preparation from washed blood cells, nitric oxide hemoglobin and sulphmoglobin. J. biol. Chem., 112: 51-65, 1935.

7. FIALON, P. et al. Aspects hématologiques du paludisme d'importation d'intérêt diagnostique dans les formes pauciparasitaires. Pathol. Biol., 39: 122-5, 1991.

8. IHEANACHO, E.N. et al. Inibition of Plasmodium falciparum growth by a synthetic iron chelator. Trans. roy. Soc. trop. Med. Hyg., 84: 213-6, 1990.

9. INTERNATIONAL NUTRITIONAL ANEMLA CONSULTATIVE GROUP. Guidelines for the eradication of iron deficiency. Goteborg, 1977.

10. LIRA, P.I.C. et al. Estado nutricional de crianças menores de seis anos, segundo posse da terra, em áreas rurais do Estado de Pernambuco, Nordeste do Brasil. Arch. latinoamer. Nutr., 35: 247-57, 1985.

11. MANTEL, N. Chi-square tests with one degree of freedom: extension of the Mantel-Haenszel procedure. J. Amer. statist. Ass., 58: 690-700, 1963.

12. McGREGOR, I.A. Malaria: nutritional implications. Rev. infect. Dis., 4: 798-804, 1982.

13. MINISTÉRIO DA SAÚDE. Superintendència de Campanhas de Saúde Pública. Divisão de Malária. Dados epidemiológicos da malária no Brasil referentes ao periodo de 1985 a 1989. Rev. Soc. bras. Med. trop, 23: 55-62, 1990.

14. MONTEIRO, C.A. \& SZARFARC, S.C. Estudo das condições de saúde das crianças no municipio de São Paulo, SP (Brasil) 1984-1985. V - Anemia. Rev. Saúde públ., S. Paulo, 22: 255-60, 1987:

15. MURRAY, M.J. et al. Refeeding malaria and hyperferraemia. Lancet, 1: 653-4, 1975.

16. MURRAY, M.J. et al. The adverse effect of iron repletion on the cause of certain infections. Brit. med. J., 2: 113-5, 1978.

17. OPPENHEIMER, S.L. Iron and malaria. Parasit. Today, 5: 77-9, 1989.

18. OPPENHEIMER, S.J. et al. Iron supplementation and malaria. Lancet, 1: 389-90, 1984.

19. ORGANIZACION MUNDIAL DE LA SALUD. Diagnostico de la malaria: memorandum de una reunión de la OMS. Bol. Ofic. sanit. panamer., 107 118-50, 1989.
20. ORGANIZACION MUNDIAL DE LA SALUD. Grupo Científico sobre Anemias Nutricionales, Ginebra, 1967. Informe. Ginebra, 1968. (Série de Informes Técnicos, 405).

21. ORGANIZACION PANAMERICANA DE LA SALUD. Manual de diagnóstico microscópico de la malária. 4a. ed. Washington, DC, 1975. (Publicación Científica, 276)

22. PIEKARSKI, G. Medical parasitology. Berlim, Springer-Verlag, 1989. p. 96-107.

23. REUNION MLXTA ADI/OIEA/OMS sobre LUCHA contra la ANEMIA NUTRICIONAL,

ESPECIALMENTE contra la CARENCIA de HIERRO, Ginebra, 1974. Informe. Ginebra, 1975. (OMS - Série de Informes Técnicos, 580).

24. SALZANO, A.C. et al. Prevalência de anemia no ciclo gestacional em dois Estados do Nordeste brasileiro, Pernambuco e Paraíba. Rev. bras. Pesq. med. biol., 13: $211-4,1980$

25. SALZANO, A.C. et al. Anemia em crianças de dois serviços de saúde de Recife, PE (Brasil). Rev. Saúde públ., S. Paulo, 19: 499-507, 1985.

26. SIGULEM, D.M. et al. Anemia ferropriva em crianças do município de São Paulo. Rev. Saúde públ., S. Paulo, 12: 168-78, 1978.

27. STEPHENSON, L.S. \& HOLLAND, C. The impact of helminth infections on human nutrition. London, Taylor \& Francis, 1987.

28. SZARFARC, S.C. Anemia ferropriva em parturientes e recém-nascidos. Rev. Saúde públ., S. Paulo, 8: 369-74, 1974.

29. SZARFARC, S.C. Anemia nutricional entre gestantes atendidas em Centros de Saúde do Estado de São Paulo (Brasil). Rev. Saúde públ., S. Paulo, 19: 450-7, 1985

30. VAZ PINTO, A. et al. A anemia da gravidez em Sobradinho, cidade satélite de Brasília, Brasil. Rev. bras. Pesq. med. biol., 8: 381-5, 1975.

31. VENTURA, F. et al. Hemoglobina: dosagem pelo método da cianometahemoglobina. Uso de solução artificial para calibração dos aparelhos colorimétricos. Rev. Hosp. Clin. Fac. Med, s. Paulo, 22: 303-4, 1967.

32. WORLD HEALTH ORGANIZATION. Malaria Action Programme. Severe and complicated malaria. Trans. roy. Soc. trop. Med. Hyg., 80 (Suppl.): 1-50, 1986.

33. WORLD malaria situation: 1986-87. Part 1. Wkly epidem. Rec., 64: 241-8, 1989.

Recebido para publicaçāo em 13/11/1991 Representado em 28/2/1992 Aprovado para publicação em 9/3/1992 